\title{
Taking Orlistat: Predicting Weight Loss over 6 Months
}

\author{
Amelia Hollywood and Jane Ogden \\ Department of Psychology, University of Surrey, Guildford, Surrey GU2 7XH, UK \\ Correspondence should be addressed to Jane Ogden, j.ogden@surrey.ac.uk
}

Received 23 September 2010; Accepted 11 October 2010

Academic Editor: A. Halpern

Copyright ( 2011 A. Hollywood and J. Ogden. This is an open access article distributed under the Creative Commons Attribution License, which permits unrestricted use, distribution, and reproduction in any medium, provided the original work is properly cited.

\begin{abstract}
This study explored the predictors of weight loss following orlistat with a focus on both baseline variables and changes in beliefs and behaviours occurring over the course of taking the drug. Patients $(n=566)$ prescribed orlistat completed a questionnaire at baseline and after 6 months concerning their weight, beliefs and behaviours. By 6 months the majority had lost some weight and showed improvements in diet. Many had also stopped taking the drug and a large minority reported using it flexibly as a lifestyle drug. Those who lost most weight showed a decrease in beliefs in a medical solution, a decrease in unhealthy eating, an increased belief in treatment control and an increased belief that the unpleasant consequences are both due to their eating behaviour and just part of the drug. When taken with fatty food orlistat causes symptoms such as anal leakage and oily stools. These may encourage some patients to focus on the behavioural aspects of their weight problem thus promoting the dietary changes needed for both short and longer term weight loss. When prescribing orlistat, clinicians should encourage patients to see the consequences as an education as a means to promote the effectiveness of this form of medical management.
\end{abstract}

\section{Introduction}

Orlistat (Xenical) is currently the only available form of prescribed obesity medication which acts on the gastrointestinal system and works by reducing fat absorption in the gut which is eliminated in bowel movements. It also blocks the availability of fat-soluble vitamins (vitamins A, D, E, and $\mathrm{K}$ ), so patients may also take a vitamin supplement $[1,2]$. Current recommendations suggest that it is used for patients who have a history of failed weight-loss attempts using behavioural methods and who can demonstrate at least $2.5 \mathrm{~kg}$ weight loss by diet and exercise in the month prior to their first prescription [1]. It is suggested that patients reduce their daily calorie intake by 500 to 1000 calories to promote weight loss, and the Dietary Guidelines for Americans recommend that dietary fat is limited to about $30 \%$ of daily calories. As a result of its impact upon fat absorption, orlistat has unpleasant side effects including liquid stools, an urgency to go to the toilet, and anal leakage which are particularly apparent following a high-fat meal as the drug causes the fat consumed to be removed from the body. Between 1998 and 2005, orlistat prescriptions rose 36fold from 17,880 to 646,700 and the total cost increased by over 35-fold. Recent years have seen an additional significant increase in the number and cost of prescriptions for orlistat [3].

Research has explored the effectiveness of orlistat compared to other drug treatments, placebo, or behaviour-focused interventions. For example, Padwal et al. [4] reported that patients taking orlistat lost $2.7 \mathrm{~kg}$ more than patients taking placebo, and Avenell et al. [5] carried out a systematic review of trials involving a combination of diets, drug therapy, exercise, and behaviour therapy and concluded that adding orlistat to a dietary intervention improved weight loss by $3.26 \mathrm{~kg}$ up to 24 months. Research also indicated that orlistat reduces cholesterol and blood pressure levels and improves glycemic control when compared to placebo [4]. Similarly, Phelan and Wadden [6] concluded from their review that adding orlistat to lifestyle modification interventions improves both weight loss and weight-loss maintenance. Furthermore, in a recent updated metaanalysis, Rucker et al. [7] synthesised the results of randomised placebo- controlled trials of approved antiobesity drugs in adults aged 18 and over for one to four years. They concluded that with the active drug treatments patients were more likely to reach $5 \%$ and $10 \%$ weight-loss thresholds 
and that weight losses for three key drugs were as follows: sibutramine: $4.2 \mathrm{~kg}$; rimonabant: $4.7 \mathrm{~kg}$, and orlistat: $2.9 \mathrm{~kg}$. Research therefore indicates that orlistat can improve weight loss if used alongside behavioural and lifestyle interventions.

There remain, however, two main problems with orlistat as a treatment for obesity. First, although evidence indicates that it can improve weight-loss outcomes, these improvements are not always substantial and there is much variability with many patients showing no improvements at all. Second, research also indicates high attrition rates with patients not adhering to their medication due to the unpleasant side effects and many stoppings taking the drug entirely or using it selectively according to the content of their diet. For example, Padwal et al. [4] concluded from their review of randomised control trials that the mean attrition rate for orlistat was 33\% (11 studies, $n=6021$ ), and Vray et al. [8] suggested that in clinical practice attrition rates are even higher at $64 \%-77 \%$. Research has therefore addressed whether the effectiveness of orlistat can be improved, and some studies have explored whether specific patients benefit more than others and whether baseline variables predict outcomes. However, whereas research exploring alternative forms of medical management has explored a range of clinical, psychological, and behavioural variables as predictors of outcomes (e.g., [9-11]), research focusing on orlistat has mainly emphasised laboratory and clinical variables [12]. In general, however, such studies conclude that the best predictor of outcome following medical management is initial weight loss, but to date few studies have explored psychological and behavioural predictors of outcome following orlistat. An alternative approach has addressed the mechanisms of how orlistat works, and from a medical perspective the main consequence of orlistat is to reduce fat absorption in the gut. However, due to the unpleasant side effects, Finer has labelled it the "antabuse effect" [13] as it deters the intake of high-fat foods. Further, Ogden and Sidhu [14] carried out a qualitative study with patients who had taken orlistat to explore their beliefs about why it either did or did not facilitate weight loss. The results showed that inline with previous research some patients stopped taking their medication due to the unpleasant symptoms such as anal leakage or oily stools. However, the results also showed that these highly visual side effects encouraged some people to consider their behaviour as a cause of their obesity. Many obese people focus on medical causes of their problem such as hormones and genetics $[15,16]$. The results from this qualitative study of orlistat users indicated that by, showing patients the fat they have consumed, orlistat can shift patient models of obesity towards a more behavioural perspective, thus encouraging them to adopt a healthier diet. Leventhal et al. [17] described the notion of coherence between beliefs about causes and solutions to any particular medical problem. Inline with this, Ogden and Sidhu [14] argued that orlistat functions by educating patients and creating coherence between behavioural causes and therefore behavioural solutions for obesity. To date, however, this process remains untested in a larger quantitative study.

In summary, although orlistat is currently the most commonly prescribed medication for the obese, there remains much variability in its effectiveness with only a minority of patients showing weight loss. Research has therefore explored the possible reasons for the effectiveness of orlistat, and whereas some studies have emphasised baseline characteristics, others have highlighted changes in beliefs and behaviour brought about by the mechanisms of the drug itself. To date, however, such studies have focused either on drugs other than orlistat, have been limited in their choice of variables, or have used small qualitative designs. The present study, therefore, aimed to explore weight loss following a 6-month course of orlistat and to explore the role of demographics, beliefs, and behaviour in predicting outcomes in a large sample of patients. Furthermore, inline with a focus on mechanisms, the study aimed to assess the role of both baseline variables and the changes occurring whilst orlistat was being taken.

\section{Method}

2.1. Design. The study used a longitudinal design with measures concerning BMI, experiences of taking orlistat, and beliefs and behaviour being completed at baseline and sixmonth followup.

2.2. Sample. Participants who had been prescribed orlistat by the GP and registered on the Xenical support system (MAP) funded by Roche were invited to take part in the study and sent the baseline questionnaire. Those who returned the baseline questionnaire were sent a further questionnaire at six months. Only those who completed the baseline questionnaire within the first three months of starting to take the medication and returned the 6 month followup questionnaire were included in this study. 566 participants returned both the baseline questionnaire within the first three months of starting to take orlistat and the 6 month followup questionnaire. This represented a response rate of $36 \%$ of total baseline responders. The University Ethics Committee approved the study. The data presented here reflect the short-term followup from baseline to six months.

2.3. Procedure. MAP gained initial consent from participants to pass on their contact details to take part in the study. An information sheet and questionnaire were then sent out to participants by post. Those who returned the baseline questionnaire were sent a similar followup questionnaire at six months.

2.4. Measures. Baseline and followup questionnaires examined demographics, beliefs about obesity, beliefs about side effects, and behaviour. All beliefs and behaviour were assessed using items which were rated using a 5-point Likert scale ranging from "not at all" (1) to "totally" (5). For each construct the individual items were summated and the reliability of each construct was assessed using Cronbach's alpha. Most alphas were above the established cutoff level illustrating acceptable reliability. Some were lower, but this is generally acceptable if there is diversity in the constructs being measured. 
(1) Demographics: participants completed measures of weight, height, BMI, age, sex, and employment.

(2) Beliefs about obesity: this included measures relating to (i) behavioural causes of obesity $(\alpha=.671)$ (e.g., "eating too much," "not enough exercise"); (ii) medical causes of obesity $(\alpha=.659)$ (e.g., "genetics/inheritance," "slow metabolism"); (iii) behavioural solutions to obesity $(\alpha=.850)$ (e.g., "eating fewer calories," "being more active"); (iv) medical solutions to obesity $(\alpha=.630)$ (e.g., "medication," "surgery"); (v) personal control over weight (e.g., "how much control do you think you have over your weight"); (vi) treatment control over weight (e.g., "how much do you think Xenical can help your weight?").

(3) Beliefs about side effects: participants rated (i) the extent to which they had experienced side effects ( $\alpha=$ .761) (e.g., "liquid stools," "bloating"), (ii) the extent to which they believed these side effects were part of the drug $(\alpha=.671)$ (e.g., "they are a necessary part of taking the drug"), and (iii) whether the side effects were caused by eating behaviour $(\alpha=.826)$ (e.g., "they have made me realise what is in different foods").

(4) Behaviour: participants rated their behaviour in terms of (i) adherence to medication $(\alpha=.577)$ (e.g., "I take it religiously," "I stop taking it before a fatty meal" (which was reverse scored)), (ii) healthy eating $(\alpha=.580)$ (e.g., healthy snacks (e.g., rice cakes, crackers, and fruit), healthy cooking (e.g., boil, steam, and cooked meals at home), and healthy food choices (e.g., skimmed milk, low-fat cheese, and high intake of fruit and vegetables) (iii) unhealthy eating ( $\alpha=$ .547) (e.g., unhealthy snacks (e.g., crisps, cakes, and chocolate), unhealthy cooking (e.g., shallow fry, deep fry, processed foods), and unhealthy food choices (e.g., full-cream milk, full-fat butter/margarine, and low intake of fruit and vegetables).

All measures related to the past month were based on previous qualitative research which has explored people's beliefs and experiences of taking obesity medication and successful weight loss and maintenance $[14,18]$. Participants also completed the personal and treatment control items of the brief Illness Perception Questionnaire that assesses participants' beliefs about their illness [19]. In addition the healthy and unhealthy eating measures were taken from the World Health Organisation 2001/02 protocol [20], the food frequency questionnaires found in the study by Inchley et al. [21], the seven-day food diary [22], and consumer marketresearch report data [23].

2.5. Inclusion/Exclusion Criteria. Participants were included if they registered with the MAP program within a fourmonth period, were 18 years or over, and had been prescribed orlistat by their GP and if they had completed the baseline questionnaire within the first three months of starting to take orlistat and also returned the followup questionnaire at six months.

2.6. Data analysis. The data were analysed to describe the participants' demographics, differences between responders and nonresponders, and overall changes in BMI, weight, and behaviour. Further, the data were analysed to assess the role of baseline demographics, beliefs, and behaviour in predicting improvements in BMI and to assess the role of changes in beliefs and behaviour over the course of 6 months in predicting improvement in BMI by 6 months.

\section{Results}

3.1. Participants' Demographics and Responders versus Nonresponders. Responders', to the questionnaire, versus nonresponders' (at 6 months) demographic variables are shown in Table 1.

The results showed that the mean age of the people who returned a completed questionnaire at both time points (responders) was 50 years and that the majority were white, female, not working, married, educated up until college, and with a mean BMI of 36 . Further, the responders and nonresponders (those who returned the baseline questionnaire but not the followup questionnaire at 6 months) were comparable on all baseline demographics apart from age with the responders being older than the nonresponders.

3.2. Overall Changes in BMI, Weight, and Behaviour. Change scores for BMI, beliefs, and behaviour were calculated (T1T2) and then classified into groups: weight loss versus no weight loss; decrease in BMI versus no decrease in BMI; improvement in healthy eating versus no improvement; increased unhealthy eating versus no increase, and for adherence those who had stopped taking it by 6 months were grouped as "nonadherers", those who rated their adherence as "totally" were rated as "adherers", and those who reported flexible adherence according to what they were eating were recoded as "lifestyle adherers." These results are shown in Table 2.

The results showed that by 6 months the majority of the responders had lost weight and decreased their BMI. The mean weight loss was $4.09 \mathrm{~kg}$ (SD: 6.21 ), the median weight loss was $3.63 \mathrm{~kg}$, and percentage weight losses were as follows: 0-2 kg: $19.36 \%$; $2.1-5 \mathrm{~kg}$ : $28.1 \% ; 5.1-7 \mathrm{~kg}$ : $15.9 \%$; 7.1-10 kg: $17.4 \% ; 10.1 \mathrm{~kg}: 19.3 \%$. In addition, the majority had increased their healthy eating and decreased their unhealthy eating and were no longer taking orlistat although a large minority reported either full adherence or being lifestyle users by 6 months.

3.3. Predictors of Improvement in BMI over 6 Months. The results were then analysed to assess the role of beliefs and behaviour in predicting an improvement in BMI by 6 months both in terms of baseline and change scores using Multiple Regression Analysis and using forced entry method. 
TABLE 1: Responders versus nonresponders at baseline.

\begin{tabular}{|c|c|c|c|c|}
\hline Variable & Responders $(n=568)$ & Nonresponders $(n=1008)$ & $t / \chi^{2}$ & $P$ \\
\hline \multirow[t]{3}{*}{ Age } & $x=50.24$ & $x=47.60$ & 14.59 & $.0001^{*}$ \\
\hline & $\mathrm{SD}=13.01$ & $\mathrm{SD}=13.11$ & & \\
\hline & $n=560$ & $n=993$ & & \\
\hline \multirow[t]{2}{*}{ Sex } & Male $=98(17.5 \%)$ & Male $=181(18.1 \%)$ & .109 & .741 \\
\hline & Female $=463(82.5 \%)$ & Female $=817(81.9 \%)$ & & \\
\hline \multirow[t]{5}{*}{ Ethnicity } & White $=543(97.1 \%)$ & White $=950(94.8 \%)$ & 4.705 & .319 \\
\hline & Black Caribbean $=4(0.7 \%)$ & Black Caribbean $=14(1.4 \%)$ & & \\
\hline & Black African = $2(0.4 \%)$ & Black African $=7(0.7 \%)$ & & \\
\hline & Asian $=5(0.9 \%)$ & Asian $=16(1.6 \%)$ & & \\
\hline & Other $=5(0.9 \%)$ & Other $=15(1.5 \%)$ & & \\
\hline \multirow[t]{3}{*}{ Job } & Full time $=174(31.6 \%)$ & Full time $=324(33.5 \%)$ & .935 & .627 \\
\hline & Part time $=113(20.5 \%)$ & Part time $=204(21.1 \%)$ & & \\
\hline & Not Working $=264(47.9 \%)$ & Not Working $=439(45.4 \%)$ & & \\
\hline \multirow[t]{5}{*}{ Marital Status } & Married $=330(59.6 \%)$ & Married = $598(61.5 \%)$ & 1.370 & .849 \\
\hline & Divorced = $66(11.9 \%)$ & Divorced = $122(12.6 \%)$ & & \\
\hline & With Partner $=64(11.6 \%)$ & With Partner $=98(10.1 \%)$ & & \\
\hline & Single $=71(12.8 \%)$ & Single $=118(12.1 \%)$ & & \\
\hline & Widowed $=23(4.2 \%)$ & Widowed = $36(3.7 \%)$ & & \\
\hline \multirow[t]{7}{*}{ Education } & $<$ secondary $=68(12.4 \%)$ & $<$ secondary $=125(13.2 \%)$ & 9.525 & .146 \\
\hline & Sec Sch Grad $=178(32.5 \%)$ & Sec Sch Grad = $332(35 \%)$ & & \\
\hline & Some Coll = $133(24.3 \%)$ & Some Coll = $239(25.2 \%)$ & & \\
\hline & Coll Grad = 78 (14.3\%) & Coll Grad = $140(14.8 \%)$ & & \\
\hline & Graduate $=54(9.9 \%)$ & Graduate $=54(5.7 \%)$ & & \\
\hline & Postgraduate $=12(2.2 \%)$ & Postgraduate $=21(2.2 \%)$ & & \\
\hline & Doct/Prof $=24(4.4 \%)$ & Doct/Prof $=37(3.9 \%)$ & & \\
\hline \multirow[t]{3}{*}{ BMI } & $x=36.20$ & $x=35.98$ & .452 & .501 \\
\hline & $\mathrm{SD}=5.84$ & $\mathrm{SD}=6.01$ & & \\
\hline & $n=527$ & $n=948$ & & \\
\hline
\end{tabular}

TABLE 2: Descriptive analysis of baseline to 6 months data.

\begin{tabular}{llll}
\hline & No & Yes & Lifestyle \\
\hline Lose weight & $n=13126.4 \%$ & $n=36573.6 \%$ \\
Decrease in BMI & Range $=-18$ to 0.07 & Range $=0.08$ to 25 \\
& $n=12826 \%$ & $n=36474 \%$ \\
Increased healthy eating & Range $=-6.15$ to 0.02 & Range $=0.03$ to 9 \\
Increased unhealthy eating & $n=18741.7 \%$ & $n=26158.3 \%$ \\
& Range $=-8$ to 0.07 & Range $=0.08$ to 13 \\
Adherence & $n=31874.1 \%$ & $n=11125.9 \%$ \\
& Range $=-8$ to 0.07 & Range $=0.08$ to 5 \\
& $n=194$ & $n=124$ \\
\end{tabular}

3.3.1. Baseline Predictors of BMI Improvement. The role of baseline beliefs and behaviour in predicting improvements in BMI are shown in Table 3.

The results showed that an improvement in BMI was predicted by a greater endorsement of a medical solution to their weight problem at baseline, accounting for 7.9\% of the variance $(F=2.862, P=.001)$. No other baseline variables were significant.
3.3.2. Changes in Beliefs and Behaviour as Predictors of BMI Improvement. Change scores in beliefs and behaviour were calculated (T1-T2). The role of these variables in predicting improvements in BMI is shown in Table 4.

The results showed that a decrease in BMI over 6 months was predicted by a decrease in endorsing a medical solution to their weight problem, a decrease in unhealthy eating, an increased belief in treatment control, and an increased belief 
TABLE 3: Baseline predictors of a decrease in BMI over 6 months.

\begin{tabular}{lcc}
\hline Variables & $\begin{array}{c}\text { Standardised } \\
\beta \text { coefficient }\end{array}$ & $P$ \\
\hline Age & .082 & .194 \\
Sex & .072 & .234 \\
Behavioural cause & -.100 & .161 \\
Medical cause & -.048 & .430 \\
Behavioural solution & .078 & .267 \\
Medical solution & .157 & $.012^{*}$ \\
Treatment control & .044 & .527 \\
Personal control & .078 & .217 \\
Experience of side effects & -.134 & .066 \\
Side effects part of the drug & -.001 & .984 \\
Side effects due to eating behaviour & -.032 & .621 \\
Healthy diet & .018 & .766 \\
Unhealthy diet & -.125 & .054 \\
\hline & & Adjusted \\
& & $R^{2}=.079$ \\
\hline
\end{tabular}

TABLE 4: Change scores as predictors of improvements in BMI over 6 months.

\begin{tabular}{lcc}
\hline Variables & $\begin{array}{c}\text { Standardised } \\
\beta \text { coefficient }\end{array}$ & $P$ \\
\hline Change in behavioural cause & .123 & .151 \\
Change in medical cause & .013 & .866 \\
Change in behavioural solution & -.004 & .963 \\
Change in medical solution & .228 & $.004^{*}$ \\
Change in treatment control & -.259 & $.001^{*}$ \\
Change in personal control & -.052 & .498 \\
Change in experience of side effects & -.020 & .810 \\
Change in side effects part of the drug & -.167 & $.039^{*}$ \\
Change in side effects due to eating & -.218 & $.005^{*}$ \\
behavior & & .115 \\
Change in healthy eating & .183 & $.017^{*}$ \\
Change in unhealthy eating & & Adjusted \\
\hline & & $R^{2}=.173$ \\
\hline
\end{tabular}

that the side effects are both due to their eating behaviour and just part of the drug, accounting for $17.3 \%$ of the variance $(F=4.015, P=.0001)$.

\section{Discussion}

The present study aimed to explore the predictors of weight loss following 6 months after being prescribed orlistat with a focus on both baseline variables and changes in beliefs and behaviour over the course of taking the drug.

The results showed that by the end of 6 months three quarters of patients reported both weight loss and a reduction in their BMI with the majority falling within the expected range inline with previous outcome studies $[4,5,7]$. The majority also reported an increase in healthy eating and a decrease in unhealthy eating which provides some support for the impact of orlistat on diet and its use as the "antabuse effect" [13]. Furthermore, just less than half had stopped taking their medication by 6 months, and a large minority reported using it flexibly in response to their dietary choices which is consistent with high attrition rates found in previous studies and the use of orlistat as a lifestyle drug $[4,8]$.

In terms of predictors of outcomes, only one baseline variable was related to a reduction in BMI by six months. In particular, the results showed that a greater endorsement of a medical solution to obesity predicts a greater reduction in BMI by followup indicating that those who have greater expectations of success for the drug show greater improvements. This finds reflection in the focus on baseline predictors reported for other forms of medical management (e.g., $[9,10]$ ) but suggests that it would be difficult to profile those patients at baseline who would most benefit from taking the drug.

The data were also analysed, however, to explore the role of changes in beliefs and behaviour over the course of taking orlistat and produced more promising results. In particular, those who lost most weight showed a decrease in beliefs in a medical solution, a decrease in unhealthy eating, an increased belief in treatment control, and an increased belief that the side effects are both due to their eating behaviour and just part of how the drug works. Therefore, it would seem that taking orlistat may encourage patients to focus on their behaviour rather than medical factors as solutions to obesity and subsequently improve their diet and that if such changes in beliefs and behaviour occur, weight loss is greater. This provides quantitative support for previous smaller-scale qualitative research [14] and indicates that the highly visual side effects of orlistat, while being unpleasant and a deterrent for some users, for others may help educate them towards a more behavioural focus on their weight problem.

There are some issues with the study which need to be addressed. First, the study did not include a control group as the study aimed to explore the predictors of outcomes after taking orlistat rather than to assess the effectiveness of this drug. The effectiveness of the drug has been explored elsewhere [4]. Second, both diet and weight were assessed using self-report rather than an objective measurement tool. This means that there may well be inaccuracies in the data as research has shown that people tend to underestimate both their weight and what they eat. However, for the present study such self-report measures represented the best means of measuring these variables in a large-scale nationwide survey as it would not have been feasible to call all participants into the clinic to collect more objective data. Third, due to the recruitment procedure, participants were generally completing the questionnaires within the first 3 months of taking orlistat. The baseline data, therefore, reflects their beliefs and experiences at the start of their course of taking orlistat but does not reflect that, either before or at the very start of this process. Future research should aim to recruit participants just before they take their first prescription of orlistat in order to gain a true baseline. Finally, the data does not show exactly when the participants 
stopped taking orlistat. However, by followup it is known whether the participants were currently taking the drug or if they had stopped within the last month. Therefore, although we do not have an exact marker of when the course of medication was stopped and therefore when its impact upon beliefs and behaviour ceased to occur, we do have an approximate measure which enables some assessment of the interrelationships between drug use and beliefs and behaviour change. Given these limitations, however, the study does provide some insights into the mechanisms of orlistat with a focus on the role of beliefs and behaviour in predicting weight loss.

\section{Conclusions}

To conclude, orlistat is currently the only prescribed obesity medication available for obese patients. Although research indicates that it can promote weight loss, there remains problems with adherence and much variability in patient outcomes. The present study aimed to explore predictors of outcomes as a means to improve its effectiveness. The results indicate that changes in beliefs and behaviour occurring throughout the course of taking orlistat are the best predictors of outcomes rather than baseline variables. Further, the results indicate that those patients, who show a shift away from a medical model of their problem towards a focus on their own behaviour and show improvements in their diet, lose more weight. These results have implications for patient management and the use of orlistat for weight loss. In particular, orlistat causes unpleasant side effects which may cause nonadherence. However, rather than conceptualising such side effects as unfortunate, they may be the very "active" ingredients necessary to bring about change in patients' behaviour. Therefore, when prescribing orlistat, clinicians should not only alert patients to the possibility of such consequences of eating high-fat foods, but also encourage them to focus and learn from them in terms of what they are eating, what this looks like when it is excluded from their bodies, and what this would do to their bodies if it had remained inside. Such an emphasis may encourage patients to see these consequences of the drug if they eat a diet high in fat, as an education, thus enabling them to take more ownership of their weight problem, in turn facilitating and promoting the changes in eating behaviour necessary for both short- and longer-term weight loss and maintenance.

\section{Acknowledgment}

The paper was in part funded by University of Surrey and Roche who provided PhD studentship for $\mathrm{AH}$.

\section{References}

[1] National Institute for Clinical Excellence, "Guidance on the use of orlistat for the treatment of obesity in adults," Technology Appraisal Guidance No.22, 2001.

[2] Royal College of Physicians of London, "Anti-obesity drugs: Guidance on appropriate prescribing and management,"
A report of the Nutrition Committee of the Royal College of Physicians of London, 2003.

[3] J. Srishanmuganathan, H. Patel, J. Car, and A. Majeed, "National trends in the use and costs of anti-obesity medications in England 1998-2005," Journal of Public Health, vol. 29, no. 2, pp. 199-202, 2007.

[4] R. Padwal, S. K. Li, and D. C. W. Lau, "Long-term pharmacotherapy for overweight and obesity: a systematic review and meta-analysis of randomized controlled trials," International Journal of Obesity, vol. 27, no. 12, pp. 1437-1446, 2003.

[5] A. Avenell, T. J. Brown, M. A. McGee et al., "What interventions should we add to weight reducing diets in adults with obesity? A systematic review of randomized controlled trials of adding drug therapy, exercise, behaviour therapy or combinations of these interventions," Journal of Human Nutrition and Dietetics, vol. 17, no. 4, pp. 293-316, 2004.

[6] S. Phelan and T. A. Wadden, "Combining behavioral and pharmacological treatments for obesity," Obesity research, vol. 10, no. 6, pp. 560-574, 2002.

[7] D. Rucker, R. Padwal, S. K. Li, C. Curioni, and D. C. W. Lau, "Long term pharmacotherapy for obesity and overweight: updated meta-analysis," British Medical Journal, vol. 335, no. 7631, pp. 1194-1199, 2007.

[8] M. Vray, J.-M. Joubert, E. Eschwège et al., "Results from the observational study EPIGRAM: management of excess weight in general practice and follow-up of patients treated with orlistat," Therapie, vol. 60, no. 1, pp. 17-24, 2005.

[9] V. Hainer, M. Kunesova, F. Bellisle et al., "Psychobehavioral and nutritional predictors of weight loss in obese women treated with sibutramine," International Journal of Obesity, vol. 29, no. 2, pp. 208-216, 2005.

[10] D. L. Hansen, A. Astrup, S. Toubro et al., "Predictors of weight loss and maintenance during 2 years of treatment by sibutramine in obesity. Results from the European multicentre STORM trial," International Journal of Obesity, vol. 25, no. 4, pp. 496-501, 2001.

[11] N. V. Dhurandhar, R. C. Blank, D. Schumacher, and R. L. Atkinson, "Initial weight loss as a predictor of response to obesity drugs," International Journal of Obesity, vol. 23, no. 12, pp. 1333-1336, 1999.

[12] J. J. G. Martínez, F. A. Ruiz, and S. D. Candil, "Baseline serum folate level may be a predictive factor of weight loss in a morbid-obesity-management programme," British Journal of Nutrition, vol. 96, no. 5, pp. 956-964, 2006.

[13] N. Finer, "Pharmacotherapy of obesity," Best Practice and Research: Clinical Endocrinology and Metabolism, vol. 16, no. 4, pp. 717-742, 2002.

[14] J. Ogden and S. Sidhu, "Adherence, behavior change, and visualization: a qualitative study of the experiences of taking an obesity medication," Journal of Psychosomatic Research, vol. 61, no. 4, pp. 545-552, 2006.

[15] J. Ogden, "The correlates of long-term weight loss: a group comparison study of obesity," International Journal of Obesity, vol. 24, no. 8, pp. 1018-1025, 2000.

[16] J. Ogden, I. Bandara, H. Cohen et al., "General practitioners' and patients' models of obesity: whose problem is it?" Patient Education and Counseling, vol. 44, no. 3, pp. 227-233, 2001.

[17] H. Leventhal, M. Diefenbach, and E. A. Leventhal, "Illness cognition: using common sense to understand treatment adherence and affect cognition interactions," Cognitive Therapy and Research, vol. 16, no. 2, pp. 143-163, 1992.

[18] J. Ogden and L. Hills, "Understanding sustained changes in behaviour: the role of life events and the process of reinvention," Health, vol. 12, pp. 419-437, 2008. 
[19] E. Broadbent, K. J. Petrie, J. Main, and J. Weinman, “The brief illness perception questionnaire," Journal of Psychosomatic Research, vol. 60, no. 6, pp. 631-637, 2006.

[20] C. Currie, O. Samdal, W. Boyce, and B. Smith, Health Behaviour in School-Aged Children: A World Health Organisation Cross-National Study. Research Protocol for the 2001/2002 Survey, Child and adolescent health research unit. University of Edinburgh, Scotland, UK, 2001.

[21] J. Inchley, J. Todd, C. Bryce, and C. Currie, "Dietary trends among Scottish schoolchildren in the 1990s," Journal of Human Nutrition and Dietetics, vol. 14, no. 3, pp. 207-216, 2001.

[22] J. Gregory, S. Lowe, C. J. Bates et al., National Diet and Nutrition Survey: Young people aged 4-18 years vol 1: Report of the Diet and Nutrition Survey, The Stationary Office, London, UK, 2000.

[23] Mintel Market Intelligence Food and Drink, "Market Intelligence UK Market Intelligence Standard Children's Snacking Habits," UK, May 2003. 


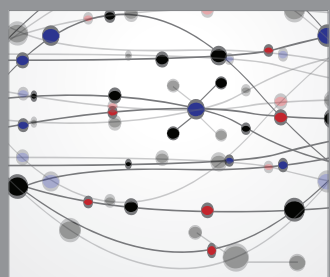

The Scientific World Journal
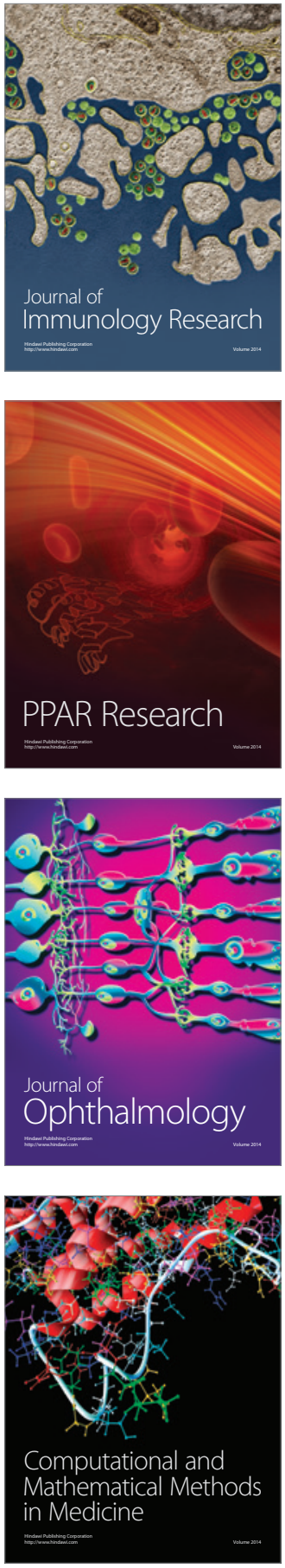

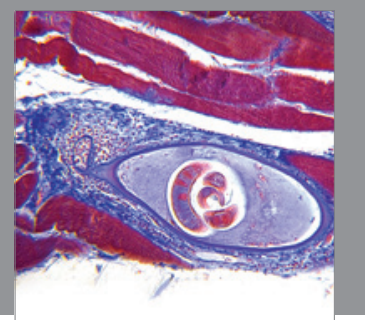

Gastroenterology

Research and Practice
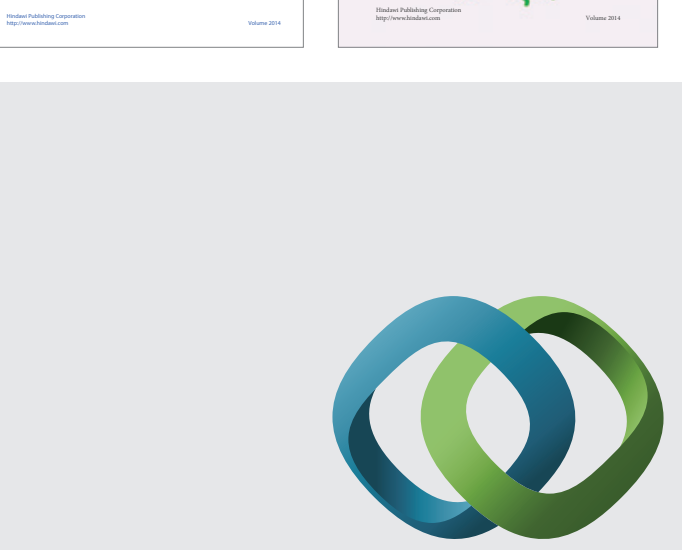

\section{Hindawi}

Submit your manuscripts at

http://www.hindawi.com
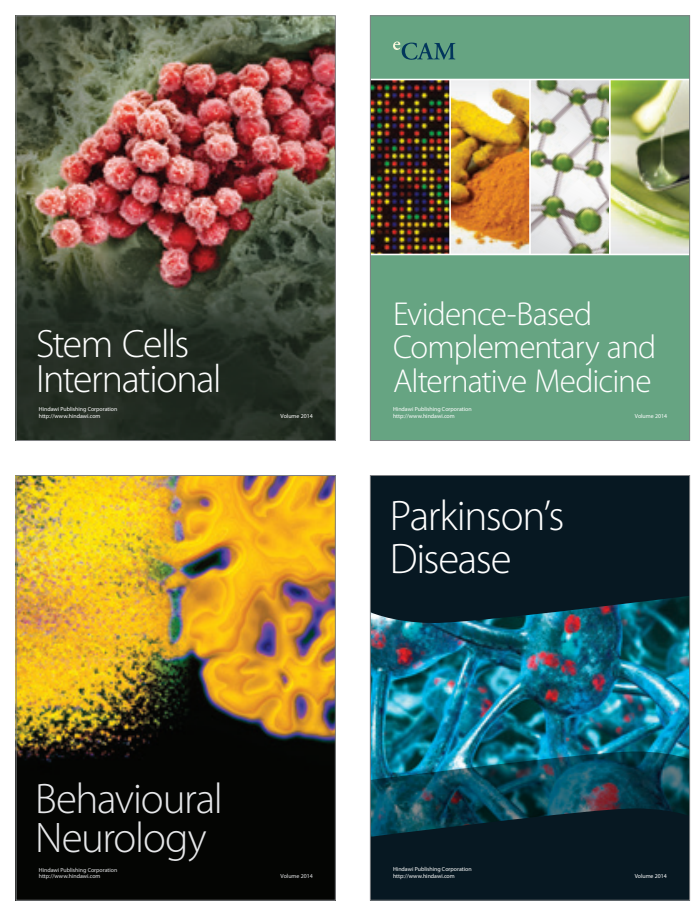

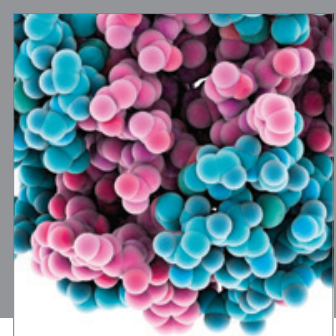

Journal of
Diabetes Research

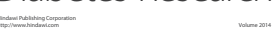

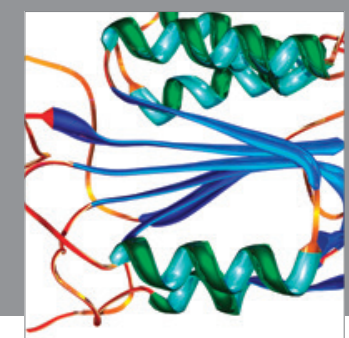

Disease Markers
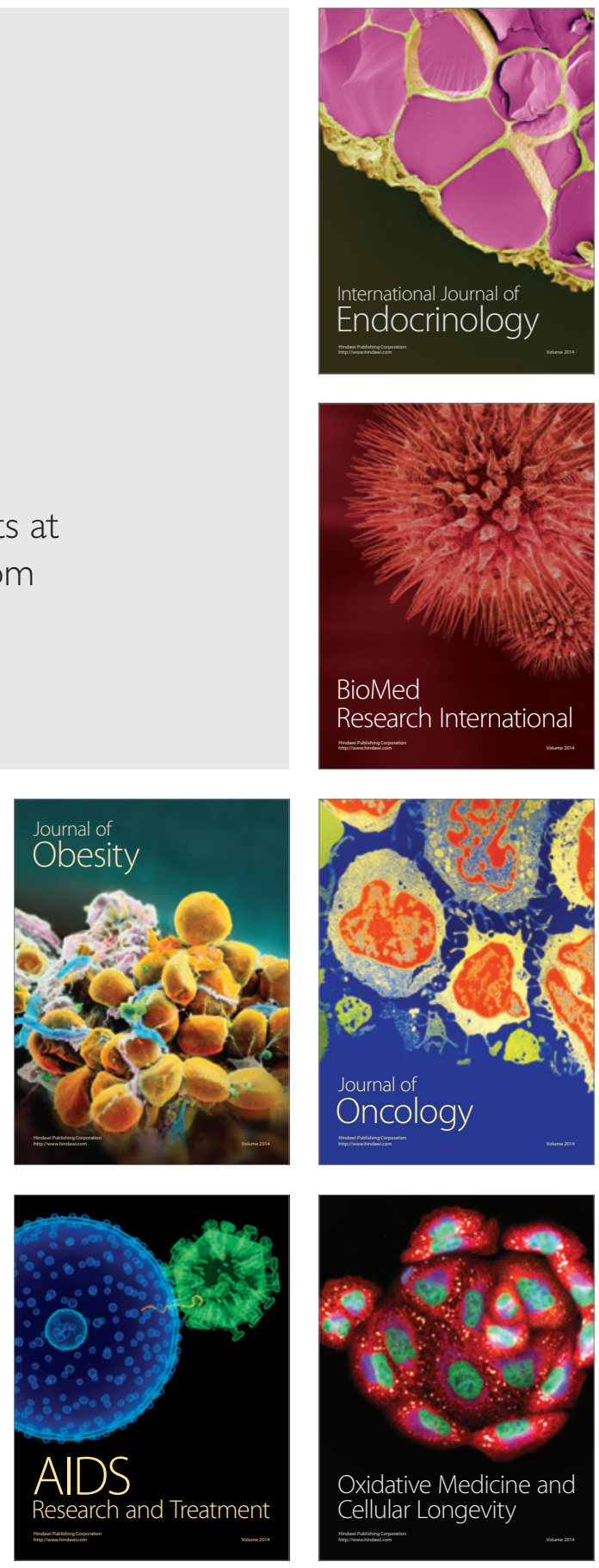